\title{
Ulcerative Colitis Following Orthotopic Cardiac Transplantation
}

\author{
Anahita Sadeghi ${ }^{1}$, Pouya Bastani ${ }^{2}$, Mehdi Mohamadnejad ${ }^{3, *}$
}

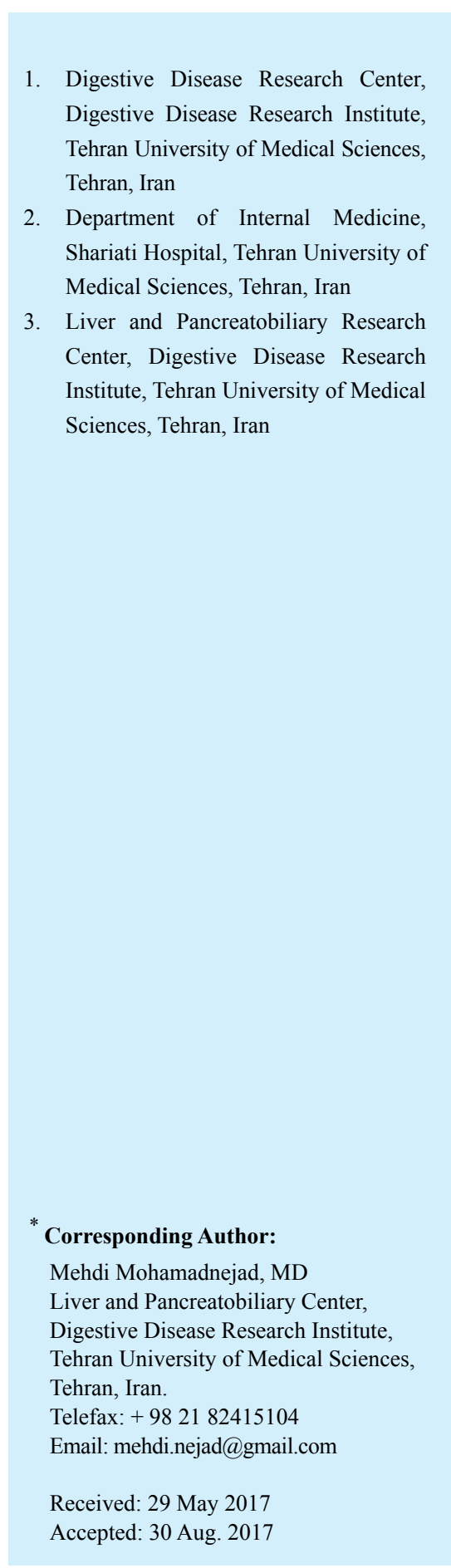

\begin{abstract}
Inflammatory bowel disease following a solid organ transplantation while the patient is receiving immunosuppressive therapy is a rare phenomenon. Here we present a 48 -year-old man who underwent cardiac transplantation 9 years earlier and was receiving cyclosporine as immunosuppressive therapy since then, presenting with complaints of rectorrhagia and diarrhea. In follow-up, he was diagnosed as having ulcerative colitis. We also reviewed the literature for similar cases, which yielded very few similar ones.
\end{abstract}

\section{KEYWORDS:}

Pancreas; Tuberculosis; Biopsy; Fine-Needle; Endosonography

Please cite this paper as:

Sadeghi A, Bastani P, Mohamadnejad M. Ulcerative Colitis Following Orthotopic Cardiac Transplantation. Middle East J Dig Dis 2017;9:235-238. doi: 10.15171/mejdd.2017.79.

\section{INTRODUCTION}

Inflammatory bowel disease (IBD), consists of two relatively different diseases, ulcerative colitis (UC) and Crohn's disease (CD) both of which have an underlying immune dysregulation. Recent studies unveil the role of specific components of cell-mediated immunity such as Th-17 in the pathogenesis of IBD regarding their role in preserving the integrity of intestinal barrier. ${ }^{1}$ Cyclosporine is an immunosuppressive agent used in a range of medical conditions notably for immunosuppressive therapy after solid organ transplantation. Whether or not cyclosporine in combination with mycophenolate mofetil (MMF) is the best combination for the patients after cardiac transplantation has been questioned in some literature suggesting the benefits of tacrolimus plus MMF over the former combination. ${ }^{2}$ Cyclosporine acts by inhibiting calcineurin, a phosphatase enzyme, which is needed for proliferation and activation of T-cells. ${ }^{3}$ Thus it would be safe to presume patients receiving cyclosporine would demonstrate lower T-cell activity. As mentioned earlier T-cells play central role in the pathogenesis of IBD, therefore for a patient receiving a combination of T-cell inhibiting agents to present with IBD would be a remote consideration.

\section{CASE REPORT}

A 48-year-old man with a history of heart transplantation 9 years earlier due to dilated cardiomyopathy was admitted with the chief compliant of chronic diarrhea with episodes of rectal bleeding for the past 3 months. He also complained of nocturnal symptoms of diarrhea and loose bloody stool. His medical history revealed a cataract surgery 


\begin{tabular}{llcc}
\multicolumn{4}{c}{ Table 1: Initial lab evaluation } \\
\hline Test & Result & Unit & Reference value \\
\hline WBC & 6420 & $1 / \mathrm{mm}^{3}$ & $4000-10,000$ \\
\hline PMN & 66 & Percent & $40-80$ \\
\hline Lymphocytes & 24 & Percent & $20-40$ \\
\hline Monocytes & 7 & Percent & $2-10$ \\
\hline Eosinophils & 3 & Percent & $1-6$ \\
\hline RBC & 3.85 & Million/mm & $4.4-6.1$ \\
\hline Hb & 11.3 & $\mathrm{~g} / \mathrm{dl}$ & $14-18$ \\
\hline Het & 34 & Percent & $42-52$ \\
\hline MCV & 88.3 & Fentoliter & $80-100$ \\
\hline MCH & 29.4 & Picogram & $26-34$ \\
\hline MCHC & 33.2 & Percent & $31-36$ \\
\hline Platelets & 145,000 & $1 / \mathrm{mm}^{3}$ & $150,000-450,000$ \\
\hline RDW & 12.6 & Percent & $11.5-14.5$ \\
\hline Cyclosporine & 247.1 & $\mathrm{ng} / \mathrm{ml}$ & $100-300$ \\
\hline Urine culture & No growth & & \\
\hline
\end{tabular}

Urine culture

No salmonella and shigella isolated

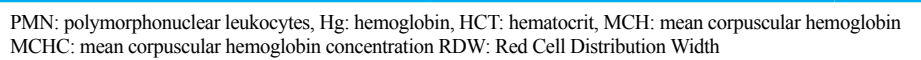

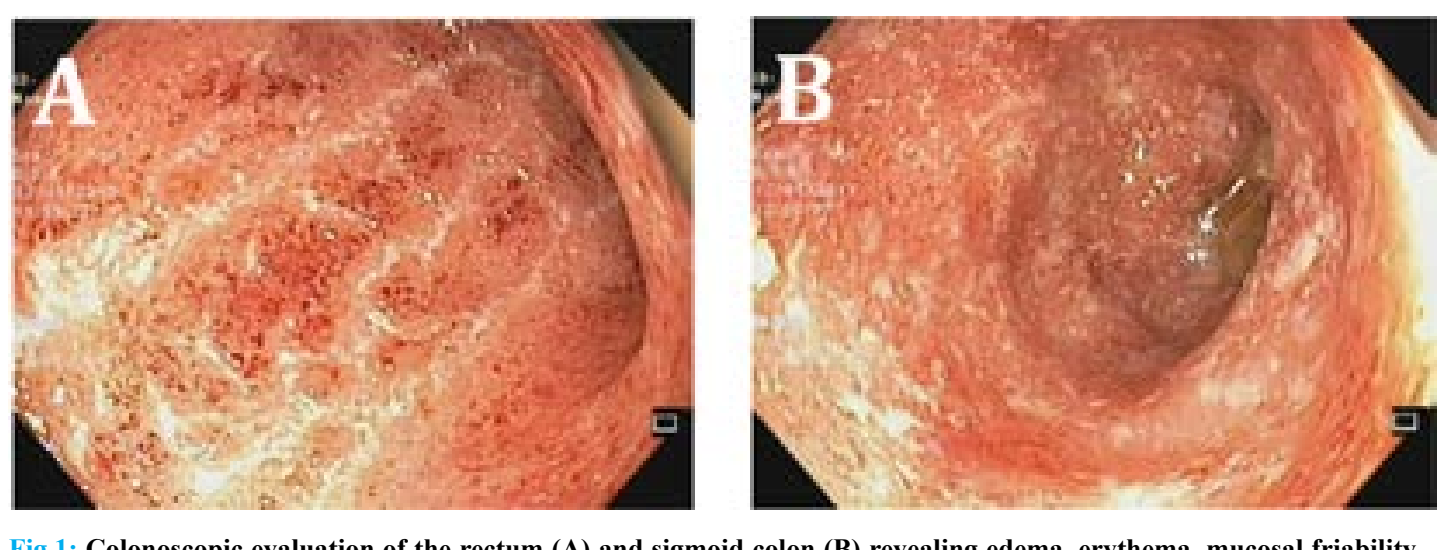

\section{Fig.1: Colonoscopic evaluation
and loss of vascular pattern.}

of the left eye, 15 years ago. He had been taking cyclosporine, initially $100 \mathrm{mg}$ daily and titrated with measuring cyclosporine level, MMF 1000 $\mathrm{mg}$ twice a day, and prednisolone $5 \mathrm{mg}$ daily since his transplantation. His other medications included: diltiazem, Inderal $₫$ (propranolol), valsartan, and atorvastatin. Initial lab data of the patient is summarized in table 1 revealing a normochromic normocytic anemia and otherwise normal results. An ileocolonoscopy was peforned. Althugh the bowel preparation was suboptimal, the visual field was improved with frequent washing and suction,

thus providing adequate quality for the procedure. The colonoscopic examination revealed edema, erythema, mucosal friability, erosions, and superficial ulcerations in rectum and sigmoid (figure 1) The descending colon, splenic flexure, transvers colon, ascending colon, cecum, and $10 \mathrm{~cm}$ of terminal ileum were all visualized with normal appearing mucosa. Several biopsy samples were taken by using biopsy forceps from the mucosa of the rectum ing bid colon, ascending colon, cecum, and terminel ileum and sent for pathological examination in Middle East Journal of Digestive Diseases/ Vol.9/No 4/October 2017
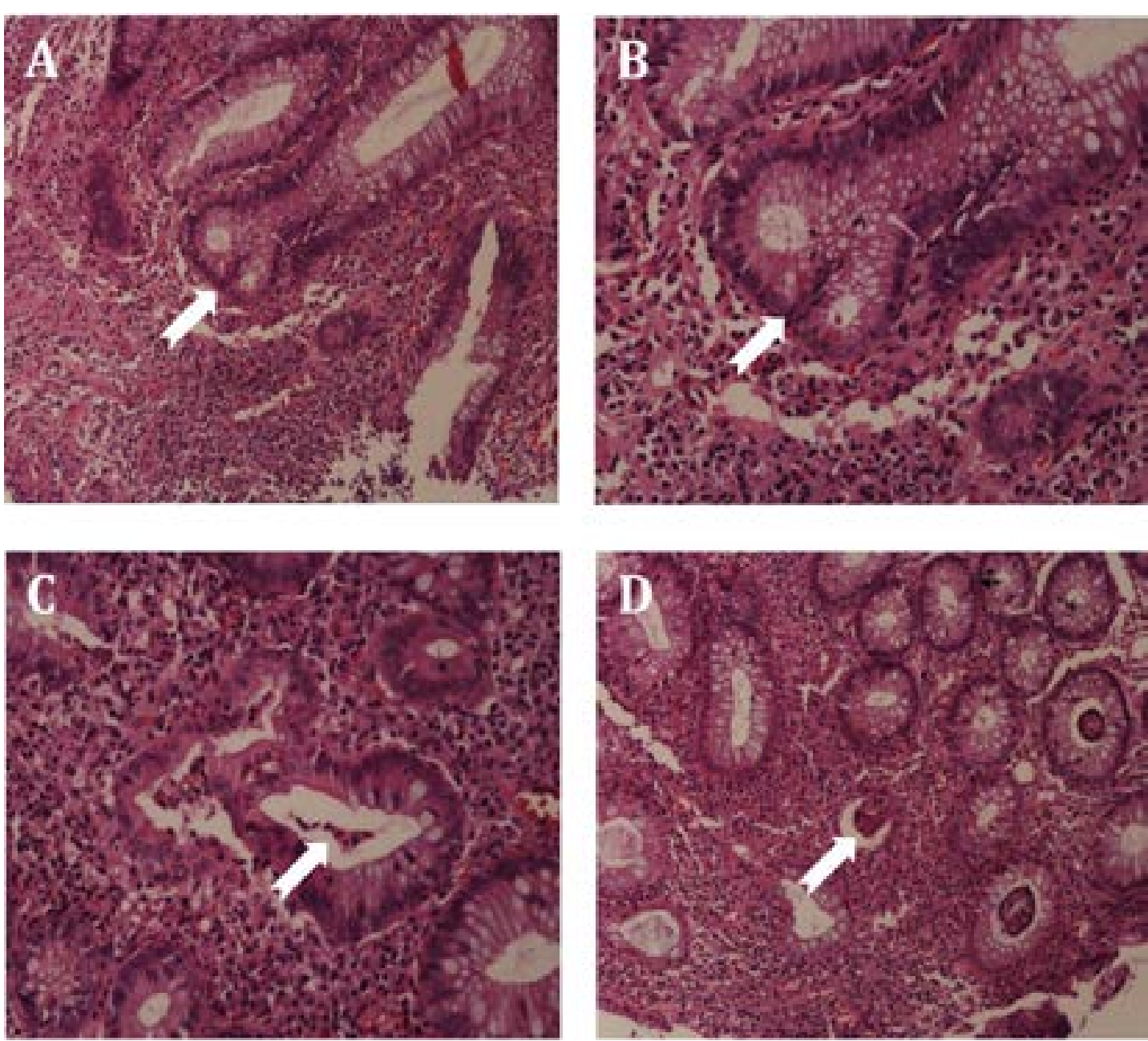

Fig.2: Sections from colonic mucosa reveal irregular sized glandular structure with branching (white arrows in figures A, and B). Lamina propria is infittrated by acute and chronic inflammatory cells with invasion into glandular structures

specimens including branching, loss of glandular architecture, and crypt abscess (figure 2) along with a negative test result for immunohistochemical (IHC) a ulcerative colitis. The patient was given mesalamine suppository, mesalamine enema, sulfasalazine, and folic acid. He responded well to the medications.

\section{DISCUSSION}

Our literature review revealed that based on studies, de novo IBD has a higher frequency in organ recipients and a more aggressive course among the mentioned poplation ${ }^{4}$ Some other cases of UC flare after solid organ ransplantant re noted. In one report a 53-year-old patient developed UC following heart transplantation de-

spite receiving cyclosporine and prednisone. Surprisingly his disease deteriorated on prednisolone, advancing from distal colitis to a pancolitis resulting in total colectomy which was finally controlled. ${ }^{5}$

There was also a patient with renal transplantation who was receiving azathioprine and prednisone. He had discontinued cyclosporine due to financial constraints and developed diarrhea, which was firstly believed to be due to immunosuppressive medication but later it was found to be a result of UC. ${ }^{6}$ In a number of patients who received orthotropic liver transplantation specially those who received transplant due to primary sclerosing cholang (PSC), UC was observed to be more conmon showed that the prevalence of UC occurring after a solid 
organ transplantation is the highest in the case of liver transplantation. ${ }^{7}$ A recent yet to be published case report, presented a patient with $\mathrm{CD}$ following cardiac transplantation who was receiving MMF. Similarly it was a remote diagnosis on the presentation considering more common causes of colitis in a patient after transplantation. It is also noted a dramatic response to treatment with anti TNF-alpha agent, infliximab, which could be promising data though some further studies are needed to confirm the efficacy and safety of infliximab in this group of patients as they pose a more complex course of disease and pathogenesis and require a multidisciplinary approach. ${ }^{4,8}$ There are also some studies, suggesting prophylactic colectomy as prevention for colorectal cancers (CRC) in selected patients with IBD/PSC who developed their disease after solid organ transplantation and pose a higher risk of CRC development. ${ }^{4}$ The case we presented along with some other cases that we reviewed, all yield a new aspect to how a disease with an immune system background can be developed during immunosuppressive therapy. These data on UC along with the development of CD in immunosuppressed patients after transplantation ${ }^{9}$ guide us to a different approach to evaluate the pathogenesis and possible etiologies. As these patients require a more complex approach to diagnosis and treatment, further studies are needed to confirm the efficacy of some novel treatments and present a clear approach to post-transplant patients with IBD.

\section{CONFLICT OF INTEREST}

The authors declare no conflict of interest related to this work.

\section{REFERENCES}

1. Da Silva BC, Lyra AC, Rocha R, Santana GO. Epidemiology, demographic characteristics and prognostic predictors of ulcerative colitis. World J Gastroenterol 2014;20:9458-67. doi: 10.3748/wjg.v20.i28.9458.

2. Guethoff S, Meiser BM, Groetzner J, Eifert S, Grinninger $C$, Ueberfuhr $P$, et al. Ten-Year Results of a Randomized Trial Comparing Tacrolimus Versus Cyclosporine A in Combination With Mycophenolate Mofetil After Heart Transplantation. Transplantation 2013;95:629-34. doi: 10.1097/ TP.0b013e318277e378.

3. Matsuda S, Koyasu S. Mechanisms of action of cyclosporine. Immunopharmacology 2000;47:119-25. doi:10.1016/S0162-3109(00)00192-2.
4. Indriolo A, Ravelli P. Clinical management of inflammatory bowel disease in the organ recipient. World J Gastroenterol 2014;20:3525-33. doi: 10.3748/wjg.v20.i13.3525.

5. Jüngling B, Kindermann I, Moser C, Püschel W, Ecker KW, Schäfers HJ, et al. Development of U1cerative Colitis after Heart Transplantation During Immunosuppressive Therapy. $Z$ Gastroenterol 2005;43:195-9. doi: 10.1055/s-2004-813743.

6. Parameswaran S, Singh K, Nada R, Rathi M, Kohli $\mathrm{H}$, Jha $\mathrm{V}$, et al. Ulcerative colitis after renal transplantation: A case report and review of literature. Indian J Nephrol 2011;21:120-2. doi: 10.4103/09714065.78063 .

7. Wörns MA, Lohse AW, Neurath MF, Croxford A, Otto G, Kreft A, et al. Five Cases of De Novo Inflammatory Bowel Disease After Orthotopic Liver Transplantation. Am J Gastroenterol 2006;101: 1931-7. doi:10.1111/j.1572-0241.2006.00624.x.

8. Noel G, Diamond B, Auerbach S, Zoeten ED, Hoffenberg E. Colonic Crohn's Disease after Cardiac Transplantation: Case Report and Literature Review. J Pediatr Gastroenterol Nutr 2017;65:e45-e46. doi: 10.1097/MPG.0000000000001069.

9. Halim MA, Said T, Nair P, Schmidt I, Hassan A, Johny $\mathrm{KV}$, et al. De Novo Crohn's Disease in a Renal Transplant Recipient. Transplant Proc 2007;39:1278-9. doi:10.1016/j.transproceed.2007.03.045. 